\title{
IL-18 haplotype GCAGT and IL-18R1 rs3755276 genotype CC combinatively associated with severity of ventilator associated pneumonia (VAP) in intensive care unit (ICU) patients
}

\section{Dunfan Chen}

Guangdong Provincial Hospital of Traditional Chinese Medicine

\section{Baijian Chen}

Guangdong Provincial Hospital of Traditional Chinese Medicine

Guowei Li

Guangdong Provincial Hospital of Traditional Chinese Medicine Haofei Wang ( $\nabla$ docflame@yeah.net)

Nanfang Hospital Affiliated to Southern Medical University

\section{Research Article}

Keywords: IL-18, haplotype, IL-18R1 rs3755276, VAP

Posted Date: May 18th, 2021

DOl: https://doi.org/10.21203/rs.3.rs-523663/v1

License: (1) (1) This work is licensed under a Creative Commons Attribution 4.0 International License.

Read Full License 


\section{Abstract}

Background: IL-18 has been reported as a collaborative biomarker for the development of sepsis surgical patients. IL-18 promoter and IL-18R1 polymorphisms have been reported in the development of postinjury sepsis and other diseases. In this study, we explored the association between IL-18/IL-18R1 polymorphisms and the severity of VAP in sepsis patients.

Methods: ELISA was used to measure the abundance of IL-18, TNF-a, IL-6 and PCT in the peripheral blood of VAP patients in distinct groups. Quantitative real-time PCR was carried out to examine the expression of IL-18 and IL-18R1 mRNA under distinct circumstances. APACHEIl scoring were performed to assess the APACHEIl scores of VAP patients with distinct genotypes.

Results: IL-18-GCAGT and IL-18R1-CC were proved to be correlated with the occurrence of SEPSIS in VAP patients. The IL-18 level was remarkably suppressed in the peripheral blood of VAP patients with IL-18GCAGT, while IL-18R1 polymorphisms showed no impact on IL-18 expression. The expression of IL-18R1 was suppressed in the peripheral blood of VAP patients with IL-18R1-CC genotypes. When combined IL-18 haplotypes and IL-18R1 genotypes together, we found IL-18-GCAGT and IL-18R1-CC were remarkably correlated with decreased APACHE II score and suppressed expression of TNF-a/IL-6/PCT and increased time from ICU admission to VAP.

Conclusion: GCAGT significantly reduced the expression of IL-18. And the genotype CC of rs 3755276 located in IL-18R1 also reduced expression of IL-18R1 compared with other genotypes. Collectively, both haplotype GCAGT of IL-18 and genotype CC of IL-18R1 were associated with severity of VAP in patients admitted to ICU.

\section{Introduction}

Sepsis is the primary cause of ventilator associated pneumonia (VAP) affecting the patients admitted in the Intensive Care Units (ICUs). About $28 \%$ of hospitalized patients who receive mechanical ventilation are prone to develop VAP leading to higher mortality rate which can reach upto $70 \%[1,2]$. Various MDR nosocomial infections drive the VAP pathophysiology in the patients who were intubated. In such conditions where all patients are more likely to being exposed to infections, investigating the host genetic involvement is complicated. The single nucleotide polymorphism plays a role in the genes involved in the host defense systems and their effects on production of cytokines and proteins [3].

Interleukin (IL) -18, a member of the IL1 cytokine superfamily, regulates immunity response, both innate and acquired, to infections. Systemic inflammatory response syndrome (SIRS) and sepsis are often a result of uncontrolled innate immune response to tissue injury. Dysregulation of cytokine as well as other inflammatory mediators' secretion cause such uncontrolled immune response. Previous findings by several groups have shown that IL-18 is involved in sepsis. Moreover, using urine levels of IL-18 in ICU patients did not successfully forecast their medical outcomes [4]. Recent evidence clearly showed that SNP in IL-18 gene along with generic variants of IL-18 gene impact the severity of disease and response 
to antiviral treatment in patients with HCV infection [5]. Two specific single nucleotide polymorphisms, $-607 \mathrm{C}>\mathrm{A}$ and $-656 \mathrm{G}>\mathrm{T}$, located on the promoter region of the IL-18 genes dictate levels of IL-18 in blood of patients with sarcoidosis. Likewise, another SNP, $+105 \mathrm{~A}>\mathrm{C}$, located on the exon 4 of IL-18 regulates expression of IL-18 in vitro [6, 7]. Binding of IL-18 to its receptor IL-18R initiates the immune response associated with it. The receptor is made up of two monomers IL-18R1 and IL-18R2; one monomer, IL18R1, controls the binding of IL-18 whereas the other monomer, IL-18R2, controls downstream signaling cascades $[8,9]$. Rs3755276, which is present on a CpG dinucleotide, is key regulator of methylation and levels of IL-18R1 mRNA. Interestingly, an inverse correlation was observed between the DNA methylation degrees and levels of mRNA.

IL-18 has been reported as a collaborative biomarker for the development of sepsis surgical patients [10]. IL-18, on the other hand, is reported to be dysregulated in septic patients, and thus demonstrated to be effective prognosis biomarker in sepsis patient recovery [11-13]. Moreover, as IL-18 promoter polymorphisms have been reported to be correlated with the development of post-injury sepsis, we suspect that IL-18 haplotype may be associated with the severity of VAP in sepsis patients [14]. Also, polymorphisms of IL-18R1 have been reported to be dysregulated in many diseases, we therefore included IL-18R1 as well as the target of this study [15-17].

\section{Materials And Methods}

\section{Human subjects and sample collection}

\section{Patients}

A retrospective study was conducted on 244 VAP patients were recruited in this study. Patients currently receiving mechanical ventilation were included in this study. The definition of VAP was based on the 2016 ATS guideline [15]. Standard bronchoalveolar lavage and aspiration were achieved. The patients were divided into two groups based on the occurrence of SEPSIS: VAP without SEPSIS and VAP with SEPSIS. The clinical features including basic demographics, such as age and sex along with underlying diseases (cardiovascular disease, pulmonary disease, cerebrovascular disease, malignancy, gastrointestinal disease and post-operation) were compared between the two groups. Institutional ethical committee of Nanfang Hospital Affiliated to Southern Medical University has approved the study protocol. Written consent forms were obtained before the initation of this study. All methods were performed in accordance with the last vision of the Declaration of Helsinki.

\section{Patient Sample Collection}

The peripheral blood samples were collected, and haplotype analysis was performed based on the polymorphisms in IL-18: G-887T, C-105T, S35S, A183G, T533C. A total of six haplotypes were defined for all of the patients: GCAAC $(\mathrm{N}=78), \operatorname{GCAGT}(\mathrm{N}=68), \operatorname{GCCAT}(\mathrm{N}=12)$, TCAAT $(\mathrm{N}=30), \operatorname{TCAAC}(\mathrm{N}=3)$, and TTCAT ( $\mathrm{N}=53)$. Furthermore, the OR were compared between VAP without SEPSIS and VAP with SEPSIS for patients with distinct haplotypes. Besides, the patients were divided into three groups based on the 
genotype of C-622T in IL18 R1: CC ( $N=161), C T(N=73)$, and TT $(\mathrm{N}=10)$. The OR were compared between VAP without SEPSIS and VAP with SEPSIS for patients with distinct genotypes at C-622T.

\section{RNA isolation and real-time PCR (IL-18, IL-18R1)}

In a sterile culture tube of $12 \times 75 \mathrm{~mm}$ size about $1 \mathrm{ml}$ TRIzol was added. Later to this tube was added frozen peripheral blood samples collected from the VAP patients. The blood sample mixture was pulverized for 20 seconds. The TRIzol solution was poured into a $1.5 \mathrm{~mL}$ Eppendorf tube and left at room temperature for $5 \mathrm{~min}$. Additionally, about $250 \mathrm{uL}$ of chloroform was added and the tube was shaken for about 15 seconds. The tube was kept at room temperature for 5 min and centrifuged at 10,000 rpm for 5 $\min$. The centrifugation segregated the mixture in three different layers, clear, aqueous top layer, white precipitated middle layer and pink organic bottom layer. The aqueous phase was pipetted out carefully so as to leave a thin layer of aqueous layer behind to prevent any contamination. Later, $550 \mathrm{uL}$ of isopropanol was added to the aqueous layer and mixed gently and left at room temperature for $5 \mathrm{~min}$. To maximize the yield of RNA the mixture was centrifuged for $30 \mathrm{~min}$. The isopropanol was removed and to the pellet was added $1 \mathrm{ml} 75 \%$ ethanol in DEPC treated with water. It was mixed gently and recentrifuged at $9000 \mathrm{rpm}$ for $5 \mathrm{~min}$. Approximately 15-25 uL of DEPC treated TE buffer to the RNA pellet.

The cocktail containing $1 \mathrm{uL}$ of RQ1, $2 \mathrm{uL}$ of reaction buffer, $6 \mathrm{uL}$ of DEPC-treated water and $0.5 \mathrm{uL}$ of RNase out was prepared. The master mix was made in proportion to the number of RNA samples being treated. About $2 \mathrm{ug}$ of RNA was added to a small tube and the volume was made to $11 \mathrm{uL}$ with DEPC treated water. In addition, $9 \mathrm{uL}$ of the previously made DNase master mix was added to bring the total volume to $20 \mathrm{uL}$. In a thermal cycler, the samples were incubated for $15 \mathrm{~min}$ at $37^{\circ} \mathrm{C}$ and later at $65^{\circ} \mathrm{C}$ for $20 \mathrm{~min}$. The samples were placed on ice later. The samples were centrifuged, and DNase treated RNA was immediately used for reverse transcription reaction.

For qRT-PCR analysis of miR-RNA, mature miRNAs were reverse transcribed using a reverse transcription assay kit (Thermo Fisher Scientific, Waltham, MA) based on the assay guidelines provided by the manufacturer on assay instruction, and the real-time PCR was carried out by using the All-in-One miRNA qRT-PCR assay kit (GeneCopoeia, Rockville, MD) based on the assay guidelines provided by the manufacturer on assay instruction. For qRT-PCR analysis of mRNAs, total RNA was reverse transcribed using specific primer pairs into cDNA with an mRNA reverse transcription assay kit (Takara, Shiga, Japan) based on the assay guidelines provided by the manufacturer on assay instruction. The expression data of miRNAs as well as mRNAs was normalized to U6. Finally, the relative expression levels of IL-18 and IL-18R1 mRNAs in each collected sample were determined using the $2^{-\Delta \Delta C t}$ method.

\section{Enzyme-linked immunosorbent assay}

Enzyme-linked immunosorbent assays were performed to measure blood levels of interleukin (IL)-18, interferon (IFN)- $\gamma$, interleukin (IL)- 6 and procalcitonin (PCT). Samples at 1/10 dilution were tested for the $18 \mathrm{kDa}$ bioactive isoform of interleukin (IL)-18 (Medical and Biological Laboratories, Nagoya, Japan), interferon (IFN)- $y$, interleukin (IL)-6 and procalcitonin (PCT) using dedicated kits according to the 
manufacturer's instructions. Briefly, $100 \mu \mathrm{L}$ peptide in the coating buffer was added to the wells of a microtiter plate and it was incubated overnight at $4{ }^{\circ} \mathrm{C}$. Later, the coating solution was removed, and the plate was washed three times using $100 \mu \mathrm{L}$ PBS-0.05\%Tween 20 . The washed were removed by flicking the plate over sink. Additional drops were removed by patting the plate over a paper towel. The remaining protein binding sites were blocked by adding $100 \mu \mathrm{L}$ of blocking buffer, $3 \%$ skim milk in PBS per well and incubate for $60 \mathrm{~min}$ at room temperature with mild shaking. Again, the plates were washed three times using $100 \mu \mathrm{L}$ PBS- $0.05 \%$ Tween 20 . Additionally, $50 \mu \mathrm{L}$ of diluted antibody were added to each well and the plate was incubated for $60 \mathrm{~min}$ at $37^{\circ} \mathrm{C}$ with gentle shaking. The plate was later washed six times with $100 \mu \mathrm{L}$ PBS- $0.05 \%$ Tween 20 . This was followed by addition of $50 \mu \mathrm{L}$ of conjugated secondary antibody, which was diluted with blocking buffer immediately before use. The plate was again incubated for 60 min at $37^{\circ} \mathrm{C}$. The plate was washed six times with $100 \mu \mathrm{L}$ PBS-0.05\% Tween 20. A mixture of acetic acid, TMB and $0.03 \%$ hydrogen peroxide with a volume ratio of $4: 1: 5$ was prepared and $50 \mu \mathrm{L}$ was added to each well. The plate was incubated in dark for half an hour at $37^{\circ} \mathrm{C}$. Upon a sufficient color development, add $100 \mu \mathrm{L}$ of stop solution to the wells. The absorbance of the plates was read at the optical density at $450 \mathrm{~nm}$ with a plate reader.

\section{Statistical analysis}

The quantitative and qualitative parameters of the studied characteristics of the population were done by Student's "t-test" (two groups) \& ANOVA (three or more groups) and chi-square test, respectively. Haploview software was used to analyse the haplotype of IL-18. Logistic regression analysis was used to evaluate the association between the variants and the phenotype. Unless otherwise specified, all results are presented as mean \pm S.E.M of 4 independent tests. Statistical evaluations were done by making use of the Student's t test in SPSS 21.0 software (IBM, Chicago, IL) and Prism 8.0 program (GraphPad, San Diego, $C A)$, and $P<0.05$ was deemed as statistically significant.

\section{Results}

\section{Haplotype and genotype analysis of VAP patient groups}

In this study, we recruited 244 VAP patients and divided them into two groups based on the occurrence of SEPSIS: VAP without SEPSIS and VAP with SEPSIS. The clinical features including basic demographics (age, male) and underlying diseases (cardiovascular disease, pulmonary disease, cerebrovascular disease, malignancy, gastrointestinal disease and post-operation) were compared between the two groups. No obvious difference was observed (Table 1). The peripheral blood samples were collected and haplotype analysis was performed based on the polymorphisms in IL-18: G-887T, C-105T, S35S, A183G, T533C. 6 haplotypes were defined for all of the patients: $\operatorname{GCAAC}(N=78), \operatorname{GCAGT}(N=68), \operatorname{GCCAT}(N=12)$, $\operatorname{TCAAT}(\mathrm{N}=30), \operatorname{TCAAC}(\mathrm{N}=3), \operatorname{TTCAT}(\mathrm{N}=53)$. The frequencies of each haplotype were shown in Table 2 . Furthermore, the OR were compared between VAP without SEPSIS and VAP with SEPSIS for patients with distinct haplotypes, among which GCAGT showed significant difference between patients with between VAP without SEPSIS and VAP with SEPSIS, whereas no obvious difference was found for other 
haplotypes. Besides, we divided the patients into three groups based on the genotype of C-622T in IL018 R1: CC $(N=161), C T(N=73), T T(N=10)$. The OR were compared between VAP without SEPSIS and VAP with SEPSIS for patients with distinct genotypes at C-622T. CT group showed remarkable difference between VAP without SEPSIS and VAP with SEPSIS, while no notable significance was observed for patients in CC and TT groups (Table 3).

Table 1

Demographic characteristics of the participants of this study

\begin{tabular}{|llll|}
\hline Characteristics & VAP without SEPSIS (N = 182) & VAP with SEPSIS (N= 62) & Pvalue \\
\hline Age, years & $82.5 \pm 8.7$ & $85.1 \pm 8.2$ & 0.773 \\
\hline Sex, male/female & $171 / 11$ & $53 / 9$ & 0.311 \\
\hline Underlying diseases & & & \\
\hline Cardiovascular disease & $58(31.9)$ & $24(38.7)$ & 0.633 \\
\hline Pulmonary disease & $158(86.8)$ & $51(82.3)$ & 1.239 \\
\hline Cerebrovascular disease & $53(29.1)$ & $20(32.3)$ & 0.489 \\
\hline Malignancy & $50(27.5)$ & $22(35.5)$ & 0.672 \\
\hline Gastrointestinal disease & $53(29.1)$ & $21(33.9)$ & 0.536 \\
\hline Post-operation & $42(23.1)$ & $18(29.0)$ & 0.040 \\
\hline
\end{tabular}

Table 2

Frequency information of IL-18 haplotype

\begin{tabular}{|lllllll|}
\hline N & G-887T & C-105T & S35S & A183G & T533C & Frequency \\
\hline 78 & G & C & A & A & C & 0.319 \\
\hline 68 & G & C & A & G & T & 0.278 \\
\hline 12 & G & C & C & A & T & 0.049 \\
\hline 30 & T & C & A & A & T & 0.123 \\
\hline 3 & T & C & A & A & C & 0.012 \\
\hline 53 & T & T & C & A & T & 0.219 \\
\hline
\end{tabular}


Table 3

logistic regression analysis of IIL-18 haplotype and IL18 R1 polymorphism

\begin{tabular}{|lllll|}
\hline Genotype & $\begin{array}{l}\text { VAP without SEPSIS } \\
(\mathrm{N}=\mathbf{1 8 2})\end{array}$ & $\begin{array}{l}\text { VAP with SEPSIS } \\
(\mathrm{N}=\mathbf{6 2})\end{array}$ & OR $(95 \% \mathrm{Cl})$ & P value \\
\hline IL-18 haplotype & & & Ref & \\
\hline GCAAC $(\mathrm{N}=78)$ & 58 & 20 & 1 & \\
\hline GCAGT $(\mathrm{N}=68)$ & 60 & 8 & $0.38(0.15-0.94)$ & 0.03 \\
\hline GCCAT $(\mathrm{N}=12)$ & 8 & 4 & $1.45(0.39-5.3)$ & 0.57 \\
\hline TCAAT $(\mathrm{N}=30)$ & 30 & 10 & $0.96(0.40-2.30)$ & 0.93 \\
\hline TCAAC $(\mathrm{N}=3)$ & 2 & 1 & $1.65(0.12-16.8)$ & 0.76 \\
\hline TTCAT $(\mathrm{N}=53)$ & 88 & 15 & $1.14(0.52-2.50)$ & 0.73 \\
\hline IL-18R1 genotype & & & Ref & \\
\hline CC $(\mathrm{N}=161)$ & 133 & 28 & 1 & 0.01 \\
\hline CT $(\mathrm{N}=73)$ & 20 & 23 & $2.18(1.15-4.14)$ & 0.32 \\
\hline TT $(\mathrm{N}=10)$ & 7 & 3 & $2.0(0.50-8.35)$ & 0. \\
\hline
\end{tabular}

\section{IL-18-GCAGT was correlated with decreased expression of IL-8 in the peripheral blood of VAP patients.}

In order to gain a deep insight into the molecular mechanisms of haplotypes on the distinct outcomes of VAP patients with or without SEPSIS, peripheral blood samples were harvested from patients in the 6 groups according to the IL-18 haplotypes (Fig. 1A). ELISA was performed to measure the abundance of IL-18 in the peripheral blood samples from patients with distinct haplotypes of IL-18. The abundance of IL-18 was significantly decreased in the peripheral blood sample from patients with GCAGT haplotype of IL-18 when compared with other haplotypes (Fig. 1B). Quantitative real time PCR was performed to examine the expression of IL-18 in the PBMCs of patients with distinct haplotypes. The expression of IL18 was significantly decreased in the peripheral blood sample from patients with GCAGT haplotype of IL18 when compared with other haplotypes (Fig. 1C). Besides, the expression of IL-18R1 mRNA was also examined using qPCR in the PBMCs of patients with distinct haplotypes. No significant difference was found for the expression of IL-18 R1 mRNA in the PBMCs of patients with distinct haplotypes (Fig. 1D).

\section{IL-18R1-CC was correlated with suppressed expression of IL-18R1 in the peripheral blood of VAP patients.}

VAP patients were partitioned into 3 groups according to the genotypes of IL-18R1 at C-622T: CC ( $N=$ 161), CT $(N=73), T T(N=10)$ (Fig. 2A). ELISA was performed to measure the abundance of IL-18 in the peripheral blood samples from patients with distinct genotypes of IL-18R1. The abundance of IL-18 showed no remarkable difference among the three groups (Fig. 2B). The expression of IL-18 mRNA in the 
PBMCs with distinct genotypes at IL-18R1 also showed no difference among the three groups (Fig. 2C). However, the expression of IL-18R1 was remarkably decreased in the PBMCs of VAP patients with CC genotype at IL-18R1 when compared with patients with CT and TT genotypes (Fig. 2D).

\section{IL-18-GCAGT/IL-18R1-CC was correlated with decreased APACHE II score, suppressed expression of TNFa/IL-6/PCT and increased time from ICU admission to VAP.}

Furthermore, we divided the VAP patients into four groups based on the haplotypes of IL-18 and genotypes of IL-18R1: 1. IL-18-GCAGT/IL-18R1-CC $(N=45)$, 2. IL-18-OTHERS/IL-18R1-CC ( $=116)$, 3.IL18-GCAGT/IL-18R1-CT/TT ( $=23)$, 4.IL-18-OTHERS/IL-18R1-CT/TT ( $=60)$. APACHE II score was examined for patients within the four groups, the APACHE II score was progressively enhanced in 1. IL-18GCAGT/IL-18R1-CC ( $\mathrm{N}=45)$, 3.IL-18-GCAGT/IL-18R1-CT/TT ( $\mathrm{N}=23)$, 2. IL-18-0THERS/IL-18R1-CC ( $=$ 116) and 4.IL-18-OTHERS/IL-18R1-CT/TT ( $=60)$ (Fig. 3A). The abundance of TNF-a, IL-6 and PCT was measured using ELISA in the four groups, the abundance of TNF-a was suppressed in IL-18-GCAGT/IL18R1-CC and activated in IL-18-OTHERS/IL-18R1-CT/TT when compared with IL-18-GCAGT/IL-18R1CT/TT and IL-18-OTHERS/IL-18R1-CC (Fig. 3B). The abundance of IL- 6 was progressively enhanced in 1. IL-18-GCAGT/IL-18R1-CC ( $N=45)$, 3.IL-18-GCAGT/IL-18R1-CT/TT ( $=23)$, 2. IL-18-OTHERS/IL-18R1-CC $(\mathrm{N}=116)$ and 4.IL-18-OTHERS/IL-18R1-CT/TT $(\mathrm{N}=60)$ (Fig. 3C). the abundance of PCT was progressively enhanced in 1. IL-18-GCAGT/IL-18R1-CC ( $=45)$, 2. IL-18-OTHERS/IL-18R1-CC ( $=116)$, 3.IL-18-GCAGT/IL-18R1-CT/TT ( $=23)$, 4.IL-18-0THERS/IL-18R1-CT/TT ( $=60)$ (Fig. 3D). Furthermore, the time from ICU admission to VAP was calculated for patients in the four groups, the time from ICU admission to VAP was gradually decreased for patients in in 1. IL-18-GCAGT/IL-18R1-CC (N = 45), 3.IL-18GCAGT/IL-18R1-CT/TT ( $=23)$, 2. IL-18-OTHERS/IL-18R1-CC $(\mathrm{N}=116)$ and 4.IL-18-OTHERS/IL-18R1$\mathrm{CT} / \mathrm{TT}(\mathrm{N}=60)(\mathrm{Fig} .3 \mathrm{E})$.

\section{Discussion}

In this study, we recruited VAP patients and divided them into six groups according to the haplotypes of IL-18. We evaluated the IL-18 in each haplotype group and found that the IL-18 level is GCAGT is significantly lower than other groups and simultaneously, GCAGT haplotype was significantly correlated with the occurrence of SEPSIS and suppressed expression of IL-18 in the peripheral blood of VAP patients. Interleukin-18 (IL-18) is a well-known gamma interferon (IFN-)-inducing factor. It stimulates secretion of gamma interferon (IFN) from natural killer (NK) cells, activated macrophages and T-cells [18]. IL-18 produces multiple phenotypic traits [19]. Biosynthesis of IL-18 involves a precursor protein called prolL-18 and is catalyzed by an enzyme cysteine protease called caspase-1 [19]. Interleukin (IL)-18 bolsters innate immunity by inducing Th-1 and Th2-driven immune responses [19, 20]. It was shown that upon IL-18 induced $\gamma$-IFN synthesis, various chemokines such as MIP-1a, MIP-1B band MCP-1 are produced [21]. This in turn recruits monocytes and macrophages at the infection site, leading to inflammation [22]. Endotoxins responsible for sepsis upregulate expression of IL-18 mRNA causing increased levels of IL-18 [23]. Previous study also showed that IL-18 is important for determination of severity and prognosis of sepsis $[24,25]$. Study by Cui et alia reported that pathology of sepsis involves 
upregulation of IL-18 and downregulation of miR-130a [26]. Therefore, in patients suffering from sepsis and thrombocytopenia, IL-18 and miR-130a play crucial role in pathophysiological process. Increased blood concentration of IL-18 in patients has been significantly correlated with trauma induced organ dysfunction, pancreatitis induced organ failure [27]. People suffering from Crohn's disease also showed higher levels of IL-18 in their blood samples [28]. In line with those previous reports about the role and effect of IL-18 in the development of sepsis or inflammatory response, the result of this study verified that the group of the patients with lowest level of IL-18 is associated with less sepsis in our patient population.

Blood plasma levels of IL-18 and clinical outcome are significantly associated with various IL-18 haplotypes. A polymorphism in a specific haplotype, GCAGT, could have protective effect as it is responsible for lowering IL-18 and reduced risk of cardiovascular death. Except the levels of IL-18, either in blood or in plaque, there is a lack of significant association between the haplotype and the reduced cardiovascular risk. However, further investigation is required to determine the role of genetic modulation of IL-18 present in the blood. A single nucleotide polymorphism, 183G allele, present on the haplotype GCAGT which is located at 3'-UTR site of the gene. Therefore, it can be involved in the translation or expression by interacting with the promoter. Previously, a plethora of studies have showed an impact of SNPs located in the $I L-18$ promoter region on transcription or biosynthesis of IL-18 by monocytes $[29,30]$. Even though these studies involved variety of haplotypes, cell lines, and SNPs, the T105 allele was continuously discovered haplotype connected with a reduced transcription. The effect was enhanced in the cells challenged with stimuli, such as LPS or PMA. Hence, there were no noticeable effects of standalone C-105T polymorphism were observed. The authors have studied previously understudied C105G p 18 haplotype and the results of this study showed lowest activity. Those information from previous literature may provide an explanation about how IL-18 haplotype of GCAGT is associated with lower level of IL-18 and further explain the less morbidity of sepsis in those patients recruited in this study. In addition, Mechanism of action of IL-18 involves binding to receptor IL18R1, which leads to the recruitment of IL18RAP and downstream signaling cascades. Therefore, besides haplotype of IL-18, we also involved the variant in the downstream signaling such as IL-18R1 in the analysis. In this study, we partitioned the VAP patients into three groups based on the genotypes of IL-18R1. CC genotype of IL-18R1 showed no correlation with the expression of IL-18 but was proved to be correlated with suppressed expression of IL-18R1 mRNA in the peripheral blood samples.

DNA methylation is a phenomenon which leads to both direct and indirect effects on the expression of genes [31, 32]. Therefore, reduced DNA methylation at CpG sites in the 3'UTR region is well-known for stimulation of expression of the genes [33]. Moreover, the role of DNA methylation in upregulation of cytokines in the subsets of Th. Animal studies showed presence of a binding site of Stat4 on the IL-18R1 locus. This protein-protein interaction is responsible for reduced DNA methylation of the region leading to upregulation of IL-18Ra in Th1 cell [34, 35]. The current study investigating peripheral blood leukocytes revealed higher levels of DNA methylation of IL-18R1 promoter. In this study, we divided VAP patients into four groups according to the haplotypes of IL-18 and genotypes of IL-18R1. IL-18-GCAGT/IL-18R1-CC was correlated with decreased APACHE II score, suppressed expression of TNFa/IL-6/PCT and increased time 
from ICU admission to VAP, while IL-18-OTHERS/IL-18R1-CT/TT was correlated with increased APACHE II score, activated expression of TNFa/IL-6/PCT and decreased time from ICU admission to VAP.

There are limitations in this study. Firstly, the samples size in this study is relatively small and only patients were recruited in this study, further study with larger scale of samples as well as heathy controls are warranted to confirm the result of this study. Secondly, only one signaling, IL-18 and IL-18R1, were involved in this study, and further study with more genes or signaling pathways as well as more phenotypes is needed to give us a more comprehensive view of the regulatory network of the VAP and sepsis.

\section{Conclusion}

The findings of this study demonstrated that among all the haplotypes of IL-18, GCAGT most significantly reduced the expression of IL-18. And the genotype CC of rs 3755276 located in IL-18R1 also reduced expression of IL-18R1 compared with other genotypes. Collectively, haplotype GCAGT of IL-18 and genotype CC of IL-18R1 combinatively associated with severity of VAP in patients admitted to ICU.

\section{Abbreviations}

IL-18: interleukin-18

IL-18R1: interleukin-18 receptor1

ICU: intensive care unit

VAP: ventilator associated pneumonia

\section{Declarations}

\section{AUTHOR CONTRIBUTIONS}

DFC BJC and HFW performed the majority of the laboratory work, statistics and data analysis and participated in writing. GWL and HFW were involved in writing. All authors conceived and designed the experiments and drafted the manuscript.

\section{DATA AVAILABILITY STATEMENT}

The data during the current study are available from the corresponding author on reasonable request. Conflict of interest

None

\section{Funding information}


NONE

\section{Ethics approval and consent to participate}

Institutional ethical committee of Nanfang Hospital Affiliated to Southern Medical University has approved the study protocol. Written consent forms were obtained before the imitation of this study. All methods were performed in accordance with the last vision of the Declaration of Helsinki.

\section{Consent for publication}

Not applicable

\section{Acknowledgements}

Not applicable

\section{References}

$1 \quad$ Hunter J, Annadurai S, Rothwell M: Diagnosis, management and prevention of ventilatorassociated pneumonia in the UK. Eur J Anaesthesiol 2007;24:971-977.

2 Vincent JL: Ventilator-associated pneumonia. J Hosp Infect 2004;57:272-280.

3 Namath A, Patterson AJ: Genetic polymorphisms in sepsis. Crit Care Clin 2009;25:835-856, x.

4 Siew ED, Ikizler TA, Gebretsadik T, Shintani A, Wickersham N, Bossert F, Peterson JF, Parikh CR, May AK, Ware LB: Elevated urinary IL-18 levels at the time of ICU admission predict adverse clinical outcomes. Clin J Am Soc Nephrol 2010;5:1497-1505.

5 Bazan JF, Timans JC, Kastelein RA: A newly defined interleukin-1? Nature 1996;379:591.

6 Thompson SR, Humphries SE: Interleukin-18 genetics and inflammatory disease susceptibility. Genes Immun 2007;8:91-99.

7 Arimitsu J, Hirano T, Higa S, Kawai M, Naka T, Ogata A, Shima Y, Fujimoto M, Yamadori T, Hagiwara K, Ohgawara T, Kuwabara Y, Kawase I, Tanaka T: IL-18 gene polymorphisms affect IL-18 production capability by monocytes. Biochem Biophys Res Commun 2006;342:1413-1416.

8 Hoshino K, Tsutsui H, Kawai T, Takeda K, Nakanishi K, Takeda Y, Akira S: Cutting edge: generation of IL-18 receptor-deficient mice: evidence for IL-1 receptor-related protein as an essential IL-18 binding receptor. J Immunol 1999;162:5041-5044.

9 Torigoe K, Ushio S, Okura T, Kobayashi S, Taniai M, Kunikata T, Murakami T, Sanou O, Kojima H, Fujii M, Ohta T, Ikeda M, Ikegami H, Kurimoto M: Purification and characterization of the human interleukin-18 receptor. J Biol Chem 1997;272:25737-25742. 
10 Zaki Mel S, Elgendy MY, El-Mashad NB, Farahat ME: IL-18 level correlates with development of sepsis in surgical patients. Immunol Invest 2007;36:403-411.

11 Wynn JL, Wilson CS, Hawiger J, Scumpia PO, Marshall AF, Liu JH, Zharkikh I, Wong HR, Lahni P, Benjamin JT, Plosa EJ, Weitkamp JH, Sherwood ER, Moldawer LL, Ungaro R, Baker HV, Lopez MC, McElroy SJ, Colliou N, Mohamadzadeh M, Moore DJ: Targeting IL-17A attenuates neonatal sepsis mortality induced by IL-18. Proc Natl Acad Sci U S A 2016;113:E2627-2635.

12 Zhu M, Rong X, Li M, Wang S: IL-18 and IL-35 in the serum of patients with sepsis thrombocytopenia and the clinical significance. Exp Ther Med 2020;19:1251-1258.

13 Wu Q, Xiao Z, Pu Y, Zhou J, Wang D, Huang Z, Hou D: Tnl and IL-18 levels are associated with prognosis of sepsis. Postgrad Med J 2019;95:240-244.

14 Stassen NA, Breit CM, Norfleet LA, Polk HC, Jr.: IL-18 promoter polymorphisms correlate with the development of post-injury sepsis. Surgery 2003;134:351-356.

15 Zhu G, Whyte MK, Vestbo J, Carlsen K, Carlsen KH, Lenney W, Silverman M, Helms P, Pillai SG: Interleukin 18 receptor 1 gene polymorphisms are associated with asthma. Eur J Hum Genet 2008;16:1083-1090.

16 Wang X, Zhu YF, Li DM, Qin Q, Wang Q, Muhali FS, Jiang WJ, Zhang JA: Polymorphisms of ST2IL18R1-IL18RAP gene cluster: a new risk for autoimmune thyroid diseases. Int J Immunogenet 2016;43:18-24.

17 Zhang J, Zheng L, Zhu D, An H, Yang Y, Liang Y, Zhao W, Ding W, Wu X: Polymorphisms in the interleukin 18 receptor 1 gene and tuberculosis susceptibility among Chinese. PLoS One 2014;9:e110734.

18 Nakanishi K, Yoshimoto T, Tsutsui H, Okamura H: Interleukin-18 regulates both Th1 and Th2 responses. Annu Rev Immunol 2001;19:423-474.

19 Nakanishi K, Yoshimoto T, Tsutsui H, Okamura H: Interleukin-18 is a unique cytokine that stimulates both Th1 and Th2 responses depending on its cytokine milieu. Cytokine Growth Factor Rev 2001;12:53-72.

20 Sims JE, Smith DE: The IL-1 family: regulators of immunity. Nat Rev Immunol 2010;10:89-102.

21 Dinarello CA, Fantuzzi G: Interleukin-18 and host defense against infection. J Infect Dis 2003;187 Suppl 2:S370-384.

22 Wu D, Murakami K, Liu N, Konishi M, Muneta Y, Inumaru S, Kokuho T, Sentsui H: Expression of equine interleukin-18 by baculovirus expression system and its biologic activity. Microbiol Immunol 2004;48:471-476. 
23 Reznikov LL, Kim SH, Westcott JY, Frishman J, Fantuzzi G, Novick D, Rubinstein M, Dinarello CA: IL-18 binding protein increases spontaneous and IL-1-induced prostaglandin production via inhibition of IFN-gamma. Proc Natl Acad Sci U S A 2000;97:2174-2179.

24 Eidt MV, Nunes FB, Pedrazza L, Caeran G, Pellegrin G, Melo DA, Possuelo L, Jost RT, Dias HB, Donadio MV, Oliveira JR: Biochemical and inflammatory aspects in patients with severe sepsis and septic shock: The predictive role of IL-18 in mortality. Clin Chim Acta 2016;453:100-106.

25 Okuhara Y, Yokoe S, Iwasaku T, Eguchi A, Nishimura K, Li W, Oboshi M, Naito Y, Mano T, Asahi M, Okamura H, Masuyama T, Hirotani S: Interleukin-18 gene deletion protects against sepsis-induced cardiac dysfunction by inhibiting PP2A activity. Int J Cardiol 2017;243:396-403.

26 Cui YL, Wang B, Gao HM, Xing YH, Li J, Li HJ, Lin Z, Wang YQ: Interleukin-18 and miR-130a in severe sepsis patients with thrombocytopenia. Patient Prefer Adherence 2016;10:313-319.

27 Rau B, Baumgart K, Paszkowski AS, Mayer JM, Beger HG: Clinical relevance of caspase-1 activated cytokines in acute pancreatitis: high correlation of serum interleukin-18 with pancreatic necrosis and systemic complications. Crit Care Med 2001;29:1556-1562.

28 Parrello T, Monteleone G, Cucchiara S, Monteleone I, Sebkova L, Doldo P, Luzza F, Pallone F: Upregulation of the IL-12 receptor beta 2 chain in Crohn's disease. J Immunol 2000;165:7234-7239.

29 Giedraitis V, He B, Huang WX, Hillert J: Cloning and mutation analysis of the human IL-18 promoter: a possible role of polymorphisms in expression regulation. J Neuroimmunol 2001;112:146-152.

30 Zhou Y, Yamaguchi E, Hizawa N, Nishimura M: Roles of functional polymorphisms in the interleukin-18 gene promoter in sarcoidosis. Sarcoidosis Vasc Diffuse Lung Dis 2005;22:105-113.

31 Hutchins AS, Mullen AC, Lee HW, Sykes KJ, High FA, Hendrich BD, Bird AP, Reiner SL: Gene silencing quantitatively controls the function of a developmental trans-activator. Mol Cell 2002;10:81-91.

32 Yano S, Ghosh P, Kusaba H, Buchholz M, Longo DL: Effect of promoter methylation on the regulation of IFN-gamma gene during in vitro differentiation of human peripheral blood T cells into a Th2 population. J Immunol 2003;171:2510-2516.

33 Reik W, Dean W, Walter J: Epigenetic reprogramming in mammalian development. Science 2001;293:1089-1093.

34 Yu Q, Chang HC, Ahyi AN, Kaplan MH: Transcription factor-dependent chromatin remodeling of II18r1 during Th1 and Th2 differentiation. J Immunol 2008;181:3346-3352.

35 Wei L, Vahedi G, Sun HW, Watford WT, Takatori H, Ramos HL, Takahashi H, Liang J, Gutierrez-Cruz G, Zang C, Peng W, O'Shea JJ, Kanno Y: Discrete roles of STAT4 and STAT6 transcription factors in 
tuning epigenetic modifications and transcription during T helper cell differentiation. Immunity 2010;32:840-851.

\section{Figures}

A

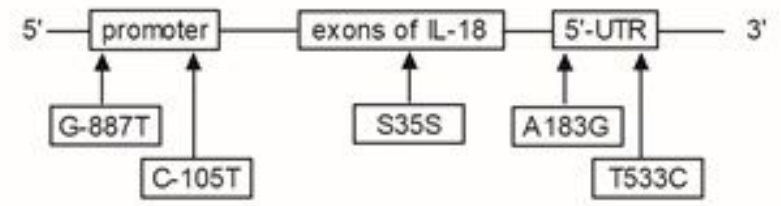

B

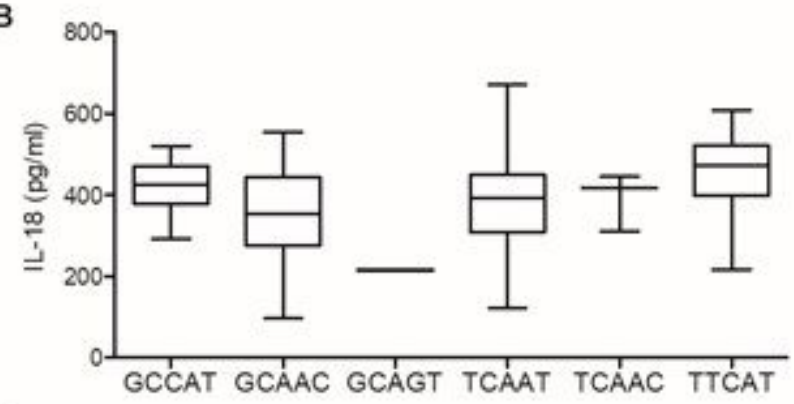

C

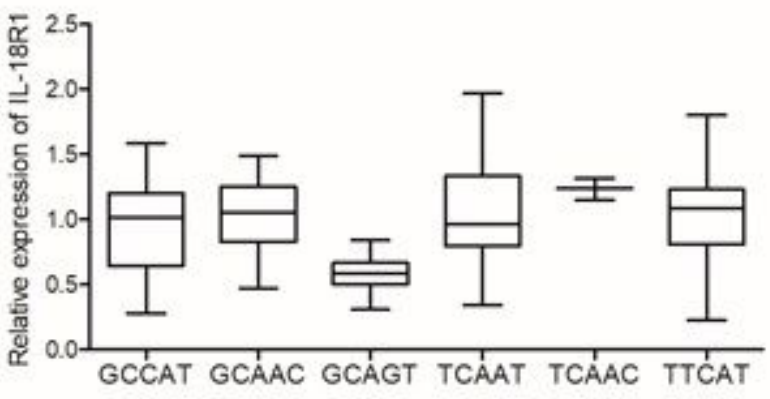

D

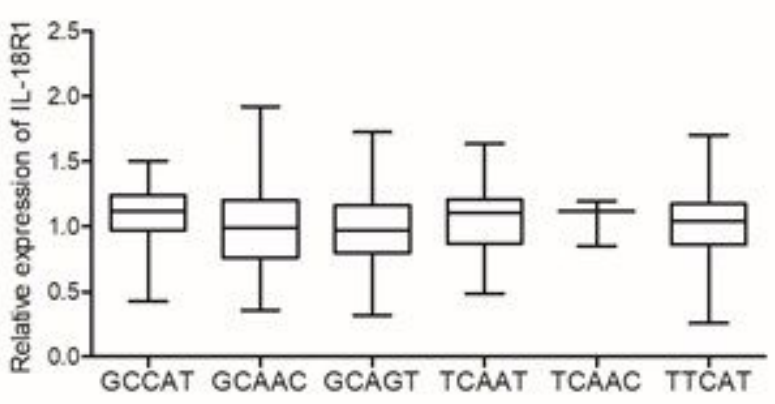

Figure 1

The expression of IL-8 was decreased in the peripheral blood of VAP patients with IL-18-GCAGT. A: Illustration of IL-18 haplotypes. B: The abundance of IL-18 was suppressed in the peripheral blood of VAP patients with IL-18-GCAGT. C: The expression of IL-18 mRNA was suppressed in the PBMCs of VAP patients with IL-18-GCAGT. D: No difference was found for the expression of IL-18R1 in the PBMCs of VAP patients with distinct haplotypes of IL-18. 
A

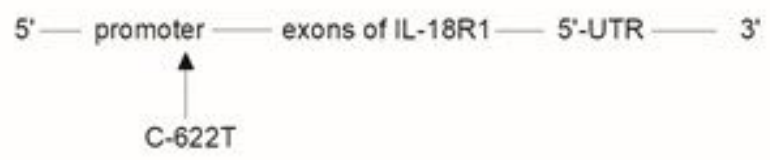

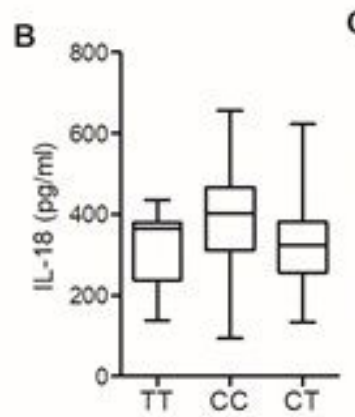

C
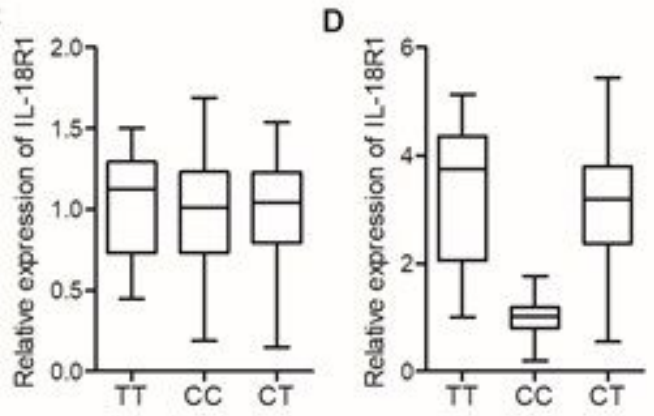

\section{Figure 2}

The expression of IL-18R1 was decreased in the peripheral blood of VAP patients with IL-18R1-CC. A:

Illustration of IL-18R1 genotypes. B: No difference was found for the abundance of IL-18 in the peripheral blood of VAP patients with distinct IL-18R1 genotypes. C: No difference was found for the expression of IL-18 mRNA in the PBMCs of VAP patients with distinct IL-18R1 genotypes. D: The expression of IL-18R1 mRNA was suppressed in the PBMCs of VAP patients with IL-18R1-CC. 

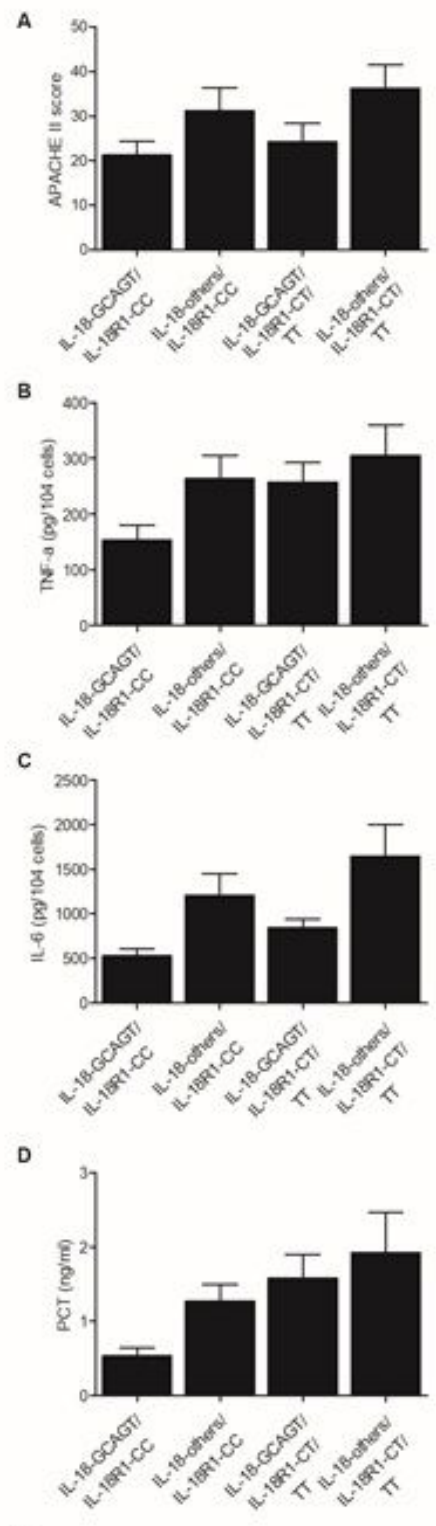

E

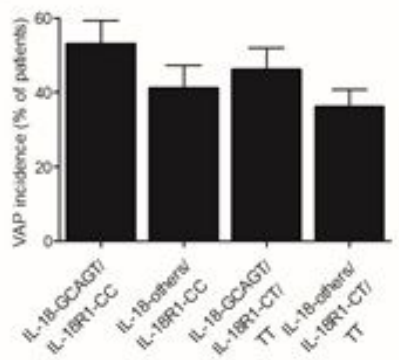

Days from ICU admissica to VAP

Figure 3

IL-18-GCAGT/IL-18R1-CC was correlated with decreased APACHE II score, suppressed expression of TNFa/IL-6/PCT and increased time from ICU admission to VAP. A: IL-18-GCAGT and IL-18R1-CC were negatively correlated with APACHEll score of VAP patients. B: The abundance of TNF-a was decreased in patients with IL-18-GCAGT/IL-18R1-CC. C: The abundance of IL-6 was decreased in patients with IL-18- 
GCAGT/IL-18R1-CC. D: The abundance of PCT was decreased in patients with IL-18-GCAGT/IL-18R1-CC. E: IL-18-GCAGT and IL-18R1-CC were correlated with increased time from ICU admission to VAP. 